\title{
Die nichtaxiomatisierbarkeit des unendlichwertigen Prädikatenkalküls von Łukasiewicz
}

\section{Journal Article}

Author(s):

Scarpellini, Bruno

Publication date:

1962

Permanent link:

https://doi.org/10.3929/ethz-b-000423097

Rights / license:

In Copyright - Non-Commercial Use Permitted

Originally published in:

The Journal of Symbolic Logic 27(2), https://doi.org/10.2307/2964111 


\section{DIE NICHTAXIOMATISIERBARKEIT \\ DES UNENDLICHWERTIGEN PRÄDIKATENKALKÜLS VON IUKASIEWICZ}

BRUNO SCARPELLINI

In dieser Arbeit soll eine unendlichwertige Logik untersucht werden, deren Aussagenkalkül von Lukasiewicz und Tarski [3] erstmals diskutiert wurde. Eine ausführliche Darstellung des Prädikatenkalküls findet man in J. D. Rutledge [6] und B. Rosser [5]. Von A. Rose und Rosser wurde die Vollständigkeit des Aussagenkalküls bewiesen [4]. Später wurde von Rutledge die Vollständigkeit des einstelligen Prädikatenkalküls bewiesen, und zwar mit algebraischen Methoden, den sogenannten $M V$-Algebras, die von C. C. Chang [1] eingeführt worden sind. Hier soll bewiesen werden, dass der volle unendlichwertige Prädikatenkalkül nicht axiomatisierbar ist. Genauer: es soll gezeigt werden, dass die Menge der wahren Formeln, d.h. derjenigen Formeln, die immer den Wert Eins annehmen, nicht rekursiv aufzählbar ist.

Beim Beweis ordnet man zuerst jeder Formel des zweiwertigen Kalküls eine solche des unendlichwertigen Kalküls zu, so dass aus der Erfüllbarkeit der ersten die Erfüllbarkeit grösser Null der Zweiten folgt und umgekehrt. Ferner versucht man, zweien Individuen, die bezüglich des zweiwertigen Kalküls als verschieden betrachtet werden müssen, zwei verschieden Werte des Intervalls $[0,1]$ zuzuordnen. Da eine unendliche beschränkte Punktmenge mindestens einen Häufungspunkt besitzt, stellt sich heraus, dass eine solche Zuordnung nur für einen endlichen Individuenbereich möglich ist. Somit lässt sich jeder Formel des zweiwertigen Kalküls eine solche des unendlichwertigen Kalküls zuordnen, dass aus der endlichen Erfüllbarkeit der Ersten die Erfüllbarkeit grösser Null der Zweiten folgt und umgekehrt. Indem man benutzt, dass die Menge der Formeln des zweiwertigen Kalküls, die nicht im Endlichen erfüllbar sind, nicht rekursiv aufzählbar ist, erhält man sofort die Aussage, dass die Menge der Formeln, die immer den Wert Null annehmen, auch nicht rekursiv aufzählbar ist. Da die Negation einer Formel, die identisch Null ist, eine solche ergibt, die immer identisch Eins ist und umgekehrt, folgt, dass auch die Menge der wahren Formeln nicht rekursiv aufzählbar ist.

Im Folgenden sollen zuerst die Formeln des unendlichwertigen Kalküls und ihre Interpretation kurz beschrieben werden.

Man hat eine abzählbare Liste $X_{m}^{1}, X_{m}^{2}, \ldots$ von Aussagenvariablen, von einstelligen Prädikatenvariablen $\phi_{m}{ }^{11}, \phi_{m}{ }^{12}, \ldots$, von zweistelligen Prädikatenvariablen $\phi_{m}^{21}, \phi_{m}^{22}, \ldots$, usw., von Individuenvariablen $x_{1}$,

Received August 26, 1960. 
$x_{2}, \ldots$ und undefinierte Terme:

Min Max $\min \max$

Man setzt fest:

(a) Eine Aussagenvariable ist eine Formel.

(b) Ist $\phi$ eine $k$-stellige Prädikatenvariable und sind $t_{1}, \ldots, t_{k}$ Individuenvariablen, so ist $\phi\left(t_{1}, \ldots, t_{k}\right)$ eine Formel.

(c) Sind $A$ und $B$ Formeln, so auch $\min (1,1-A+B)$ und $(1-A)$.

(d) Ist $A$ eine Formel, und $x$ eine Individuenvariable, so sind auch $(\operatorname{Min} A)$ und $(\operatorname{Max} A)$ Formeln.

Bezügliche der Quantoren Min und Max kann man wieder zwischen freiem und gebundenem Auftreten einer Individuenvariablen unterscheiden, und aus der Menge der oben definierten Formeln speziell die Teilmenge derjenigen betrachten, in denen eine Individuenvariable nur frei oder nur gebunden vorkommt. Hier sollen ausschliesslich Formeln betrachtet werden, die dieser letzten Teilmenge angehören (was keine Einschränkung bedeutet).

Nun seien $\varphi_{1}, \ldots, \varphi_{i}, \ldots, \varphi_{p}$ je $n_{1}, \ldots, n_{i}, \ldots, n_{p}$-stellige Prädikatenvariablen, $Y_{1}, \ldots, Y_{s}$ Aussagenvariablen. $B$ sei eine Menge von Individuen, ein Individuenbereich. $f_{1}, \ldots, f_{i}, \ldots, f_{p}$ seien $n_{1}, \ldots, n_{i}, \ldots, n_{p}$-stellige Funktionen, die auf ganz $B$ definiert sind und Werte aus $[0,1]$ annehmen. $\xi_{1}, \ldots, \xi_{s}$ sollen $Z$ ahlen aus $[0,1]$ sein.

Jetzt betrachten wir diejenigen Formeln $A$, die nur Prädikatenvariablen aus der Reihe $\varphi_{1}, \ldots, \varphi_{i}, \ldots, \varphi_{p}$ und Aussagenvariablen aus der Reihe $Y_{1}, \ldots, Y_{s}$ enthalten. Jeder solchen Formel $A$ und jeder Wahl von Individuen $\beta_{1}, \ldots, \beta_{k}$ für ihre freien Individuenvariablen $t_{1}, \ldots, t_{k}$ ordnen wir eine Zahl aus $[0,1]$ zu. Diese Zuordnung geschieht durch Induktion.

Ist $A$ eine Aussagenvariable $Y_{\boldsymbol{t}}$, so sei der Wert von $A$ gleich $\xi_{i}$.

Ist $A$ von der Form $\varphi_{i}\left(t_{1}, \ldots, t_{k}\right)$, und wurde der Individuenvariablen $t_{\imath}$ das Element $\beta_{i}$ zugeordnet, so sei der Wert von $A$ gleich $f_{i}\left(\beta_{1}, \ldots, \beta_{k}\right)$.

Ist $A$ von der Form $1-C$, und ist $c$ der Wert von $C$, so sei der Wert von $A$ die $Z$ ahl $1-c$.

Ist $A$ von der Form min $(1,1-C+D)$, ist $c$ der Wert von $C, d$ der Wert von $D$, so sei der Wert von $A$ die kleinere der beiden Zahlen 1 , $1-c+d$.

Ist $A$ von der Form $\left(\operatorname{Max}_{x} C(x)\right)$ (bezw. (Min $\left.C(x)\right)$ ), so sei der Wert von $A$ gleich limsup $C(\beta)$ (bezw. liminf $C(\beta)$ ). Eine Formel heisst identisch Null, wenn jede Bewertung ihr $\stackrel{\beta}{\boldsymbol{\beta}}$ den Wert Null erteilt. Im Folgenden soll gezeigt werden, dass die Menge der Formeln identisch Null nicht rekursiv aufzählbar ist.

Aussagenformeln sind solche, die keine Quantoren und Prädikatenvariablen enthalten. Es lässt sich zeigen [4], dass zu den Funktionen

$$
\min (x, y), \quad \max (x, y), \quad \min (1,2 x), \quad \max (x-y, 0)
$$


Aussagenformeln existieren, welche interpretiert eben diese Funktionen ergeben. Diese Aussagenformeln bezeichnen wir der Reihe nach mit

$$
(X \wedge Y), \quad(X \vee Y), \quad E(X), \quad(X \doteq Y) .
$$

Da im Folgenden der gewöhnliche, zweiwertige Prädikatenkalkül eine gewisse Rolle spielen wird, sollen seine Aussagenvariablen und Prädikatenvariablen einen untern Index 2 erhalten, um sie von den entsprechenden Variablen des unendlichwertigen Kalküls zu unterscheiden. Die Individuenvariablen sollen in beiden Kalkülen die gleichen sein, da sie im Folgenden über den gleichen Individuenbereich laufen werden. Dagegen sollen auch Bezeichnungen von Formeln durch Indizes 2 bzw. $m$ charakterisiert werden; z.B. ist $F_{2}$ die Bezeichnung einer Formel des zweiwertigen Kalküls, $F_{m}$ die Bezeichnung einer Formel des unendlichwertigen Kalküls.

Nun seien die Prädikatenvariablen des zweiwertigen Kalküls nach bekannter Art abgezählt, und die gleiche Art der Abzählung wählen wir auch für die Prädikatenvariablen des unendlichwertigen Kalküls (Aussagenvariablen sollen als nullstellige Prädikatenvariablen betrachtet werden). Ist dann $\varphi_{2}{ }^{k}$ die $k$-te Prädikatenvariable der ersten Liste, und hat $\varphi_{2}{ }^{k}$ die Stellenzahl $n_{k}$, so hat auch die $k$-te Prädikatenvariable der zweiten Liste die Stellenzahl $n_{\boldsymbol{k}}$.

Schliesslich seien auch die Individuenvariablen, die ja für beide Kalküle die gleichen sind, abgezählt.

Jetzt soll jeder Formel des zweiwertigen Kalküls auf eine noch zu beschreibende Art eine Formel des unendlichwertigen Kalküls zugeordnet werden. Diese Zuordnung wird durch Rekursion definiert.

Sei $F_{2}\left(\varphi_{2}{ }^{\alpha_{1}}, \ldots, \varphi_{2}{ }^{\alpha_{k}}\right)$ eine Formel des zweiwertigen Kalküls. $\varphi_{2}{ }^{\alpha_{1}}$ sei diejenige Prädikatenvariable, welche in der oben angegebenen Nummerierung die Nummer $\alpha_{i}$ erhält. Dann sei dem $\varphi_{2}{ }^{\alpha_{i}}$ die Prädikatenvariable $\varphi_{m}{ }^{\alpha_{i}}$ zugeordnet, d.h. diejenige Prädikatenvariable des unendlichwertigen Kalküls, welche auch die Nummer $\alpha_{i}$ hat. $x$ sei eine in $F_{2}$ auftretende Individuenvariable. Diese sei sich selbst zugeordnet (es sei bemerkt, dass die Individuenvariablen in beiden Kalkülen dieselben sind). $\Lambda_{m}$ sei die erste nullstellige Prädikatenvariable, welche in der Reihe $\varphi_{m}{ }^{\alpha_{1}}, \ldots, \varphi_{m}{ }^{\alpha_{k}}$ nicht vorkommt.

Ist dann $A_{2}$ eine Formel, die als Bestandteil von $F_{2}$ auftritt, und ist für $A_{2}$ die Zuordnung schon definiert, nämlich $A_{m}$, so soll dem $\neg A_{2}$ die Formel $E\left(\Lambda_{m}\right) \doteq A_{m}$ zugeordnet werden.

Ist ferner dem $B_{2}$ das $B_{m}$ zugeordnet, so soll dem $A_{2} \wedge B_{2}$ das $A_{m} \wedge B_{m}$ zugeordnet werden, $\operatorname{dem} A_{2} \vee B_{2}$ das $A_{m} \vee B_{m}(\neg, \wedge, \vee$ sollen im zweiwertigen wie üblich als Negation, Konjunktion und Disjunktion gedeutet werden).

Enthält $A_{2}$ die freie Individuenvariable $x$, so soll dem $(E x) A_{2} \operatorname{das} \operatorname{Max} A_{m}$ zugeordnet worden, dem $(x) A_{2}$ das $\operatorname{Min} A_{m}$. 
Damit ist jedem $F_{2}$ eindeutig und effektiv ein $F_{m}$ zugeordnet.

Jetzt sollen zwei Hilfsformeln angegeben werden, die nachher gebraucht werden. Sei wieder $F_{2}\left\langle\varphi_{2} \alpha^{\alpha_{1}}, \ldots, \varphi_{2}{ }^{\alpha_{k}}\right)$ eine Formel des zweiwertigen Kalküls und $F_{m}\left(\varphi_{m}{ }^{\alpha_{1}}, \ldots, \varphi_{m}{ }^{\alpha_{k}}, \Lambda_{m}\right)$ die nach dem obigen Verfahren zugeordnete des unendlichwertigen Kalküls. Sei $n$ die Stellenzahl von $\varphi_{m}{ }^{\alpha_{i}}$. Dann bilden wir den Ausdruck

$$
\begin{aligned}
& \operatorname{Min}_{x_{1}} \ldots \operatorname{Min}_{x_{n}}\left\{\varphi_{m}^{\alpha_{i}}\left(x_{1}, \ldots, x_{n}\right) \wedge\left(E\left(\Lambda_{m}\right)-\varphi_{m}{ }^{\alpha_{i}}\left(x_{1}, \ldots, x_{n}\right)\right) \wedge\right. \\
& \wedge\left.\wedge\left(\left(\varphi_{m}^{\alpha_{i}}\left(x_{1}, \ldots, x_{n}\right)-\Lambda_{m}\right) \vee\left(\Lambda_{m}-\varphi_{m}^{\alpha_{i}}\left(x_{1}, \ldots, x_{n}\right)\right)\right)\right\} .
\end{aligned}
$$

Für diesen führen wir die Abkürzung $\Delta\left(\varphi_{m}{ }^{\alpha_{i}}, \Lambda_{m}\right)$ und bilden die Formel

$$
\Lambda_{m} \wedge\left(1-E\left(\Lambda_{m}\right)\right) \wedge \Delta\left(\varphi_{m}^{\alpha_{1}}, \Lambda_{m}\right) \wedge \ldots \wedge \Lambda\left(\varphi_{m} \alpha_{k}, \Lambda_{m}\right) .
$$

Dies ist die erste der beiden Hilfsformeln und sie sei abgekürzt mit $K_{m}\left(\varphi_{m}, \Lambda_{m}\right)$ bezeichnet. Es sei noch kurz auf einen Umstand aufmerksam gemacht. Eigentlich müsste man zwischen $\left(\left(A_{m} \wedge B_{m}\right) \wedge C_{m}\right)$ und $\left(A_{m} \wedge\left(B_{m} \wedge C_{m}\right)\right)$ unterscheiden. Wie leicht zu sehen, stimmen aber bei jeder Bewertung die Zahlwerte der beiden Formeln überein, weshalb die Klammern in solchen Fällen weggelassen werden sollen.

Nun sei noch die zweite Hilfsformel angegeben.

Um zu dieser zu gelangen, brauchen wir den Ausdruck

$$
(X \wedge Y) \vee\left(\left(E\left(\Lambda_{m}\right)-X\right) \wedge\left(E\left(\Lambda_{m}\right)-Y\right)\right),
$$

für den wir die Abkürzung $X * Y$ einführen. Jetzt bilden wir die Formel

$$
\begin{aligned}
\operatorname{Min}_{x_{3}} \ldots \operatorname{Min}_{x_{n}}\left\{\left(\varphi _ { m } \alpha _ { i } \left(x, x_{2},\right.\right.\right. & \left.\left.\ldots, x_{n}\right) * \varphi_{m}^{\alpha_{i}}\left(y, x_{2}, \ldots, x_{n}\right)\right) \\
\wedge & \left(\varphi_{m}^{\alpha_{i}}\left(x_{2}, x, x_{3}, \ldots, x_{n}\right) * \varphi_{m}^{\alpha_{i}}\left(x_{2}, y, x_{3}, \ldots, x_{n}\right)\right) \\
& \left.\cdot \cdot \cdot \cdot \cdot \cdot \cdot\left(\varphi_{m}^{\alpha_{i}}\left(x_{2}, x_{3}, \ldots, x_{n}, x\right) * \varphi_{m}^{\alpha_{i}}\left(x_{2}, x_{3}, \ldots, x_{n}, y\right)\right)\right\},
\end{aligned}
$$

und diese sei mit $D_{m}\left(\varphi_{m} \alpha_{4}, x, y, \Lambda_{m}\right)$ abgekürzt. Nun sei $\mathscr{H}_{m}$ die erste einstellige Prädikatenvariable, die nicht unter den $\varphi_{m}{ }^{\alpha_{1}}, \ldots, \varphi_{m}{ }^{\alpha_{k}}$ vorkommt. Indem wir die Abkürzung

$$
\left|\mathscr{H}_{m}(x)-\mathscr{H}_{m}(y)\right|
$$

für

$$
\left(\mathscr{H}_{m}(x) \doteq \mathscr{H}_{m}(y)\right) \vee\left(\mathscr{H}_{m}(y)-\mathscr{H}_{m}(x)\right)
$$

einführen, bilden wir die Formel

$$
\begin{aligned}
& \underset{x}{\operatorname{Min}} \operatorname{Min}_{y}\left\{\left(\left(D_{m}\left(\varphi_{m}^{\alpha_{1}}, x, y, \Lambda_{m}\right) \wedge \ldots \wedge D_{m}\left(\varphi_{m}^{\alpha_{k}}, x, y, \Lambda_{m}\right)\right)-\Lambda_{m}\right)\right. \\
& \left.\checkmark\left|\mathscr{H}_{m}(x)-\mathscr{H}_{m}(y)\right|\right\},
\end{aligned}
$$

die wir mit $H_{m}\left(\varphi_{m}, \Lambda_{m}, \mathscr{H}_{m}\right)$ bezeichnen. Dies ist die zweite Hilfsformel. 
Nach diesen formalen Vorbereitungen soll jetzt der Zweck dieser Definitionen geklärt werden. Zuerst sei $K_{m}\left(\varphi_{m}, \Lambda_{m}\right)$ betrachtet.

Es sei $B, f_{m}^{1}, \ldots, f_{m}^{i}, \ldots, f_{m}^{k}, \lambda_{m}$ eine Bewertung derart, dass $K_{m}\left(f_{m}, \lambda_{m}\right) \geqq \delta>0$ ist. Aus der Definition von $K_{m}\left(\varphi_{m}, \Lambda_{m}\right)$ folgt

$$
\min \left(\lambda_{m}, 1-\min \left(1,2 \lambda_{m}\right), \Delta\left(f_{m}^{1}, \lambda_{m}\right), \ldots, \Delta\left(f_{m}^{k}, \lambda_{m}\right)\right) \geqq \delta>0,
$$

also

$$
\begin{gathered}
\lambda_{m} \geqq \delta>0, \\
1-\min \left(1,2 \lambda_{m}\right) \geqq \delta>0, \\
\Delta\left(f_{m}{ }^{1}, \lambda_{m}\right) \geqq \delta>0, \\
\cdot \cdot \cdot \cdot \\
\Delta\left(f_{m}^{k}, \lambda_{m}\right) \geqq \delta>0 .
\end{gathered}
$$

Aus der zweiten Ungleichung ergibt sich

$$
0<\lambda_{m}<2 \lambda_{m}<1 \text {. }
$$

Ferner gilt, auf Grund der Definition von $\Delta\left(f_{m} i, \lambda_{m}\right)$, für alle $i$

$$
\begin{aligned}
& \liminf \min \left(f_{m}^{i}\left(x_{1}, \ldots, x_{n}\right), 2 \lambda_{m}-f_{m}^{i}\left(x_{1}, \ldots, x_{n}\right)\right. \text {, } \\
& x_{1}, \ldots, x_{n} \\
& \left.\max \left(f_{m}^{i}\left(x_{1}, \ldots, x_{n}\right)-\lambda_{m}, \lambda_{m}-f^{i} m_{m}\left(x_{1}, \ldots, x_{n}\right)\right)\right) \geqq \delta>0 .
\end{aligned}
$$

D.h., für alle $n$-tupel von Elementen $\beta_{1}, \ldots, \beta_{n}$ aus $B$ liegt der Wert von $f_{m}^{\imath}\left(\beta_{1}, \ldots, \beta_{n}\right)$ in einem der beiden Intervalle

$$
I_{1}:\left[\delta, \lambda_{m}-\delta\right], \quad I_{2}:\left[\lambda_{m}+\delta, 2 \lambda_{m}-\delta\right]
$$

(man sieht sofort, dass $\delta$ den Ungleichungen $\lambda_{m} \geqq 2 \delta, 1-2 \lambda_{m} \geqq \delta$, $\frac{1}{5} \geqq \delta$ genügen muss). Hat man umgekehrt die $f_{m}$ so gewählt, dass ihre Werte in zwei Intervallen $\left[\delta, \lambda_{m}-\delta\right],\left[\delta+\lambda_{m}, 2 \lambda_{m}-\delta\right]$ liegen, wobei $\lambda_{m} \geqq 2 \delta>0,1-2 \lambda_{m} \geqq \delta, 0<\lambda_{m}<2 \lambda_{m}<1$ ist, so ist

$$
K_{m}\left(f_{m}, \lambda_{m}\right) \geqq \delta>0 \text {. }
$$

Hier sei eine Bezeichnung eingeführt, die im Folgenden nützlich sein wird. $F_{2}\left(\varphi_{2}^{\alpha_{1}}, \ldots, \varphi_{2}^{\alpha_{k}}\right)$ sei eine Formel des zweiwertigen Kalküls, $F_{m}\left(\varphi_{m}{ }^{\alpha_{1}}, \ldots, \varphi_{m}{ }^{\alpha_{k}}, \Lambda_{m}\right)$ sei die zugeordnete des unendlichwertigen Kalküls. Wir sagen von einer Formel $F_{m}{ }^{\prime}$, sie sei Bild von $F_{2}$, wenn sie aus $F_{m}\left(\varphi_{m}^{\alpha_{1}}, \ldots, \varphi_{m}{ }^{\alpha_{k}}, \Lambda_{m}\right)$ durch Ersetzen von $\Lambda_{m}$ durch $\Lambda_{m}^{\prime}$ hervorgegangen ist, wobei $\Lambda_{m}{ }^{\prime}$ eine Aussagenvariable ist, die nicht in der Reihe $\varphi_{m}{ }^{\alpha_{1}}, \ldots$, $\varphi_{m}{ }^{\alpha_{k}}$ vorkommt. Ist z.B. $F_{2}$ von $\operatorname{der}$ Form $A_{2} \wedge B_{2}$, so ist $F_{m}$ von der Form $A_{m} \wedge B_{m}$, wobei $A_{m}$ Bild ist von $A_{2}, B_{m}$ Bild von $B_{2}$. Es gilt der:

Hilfssatz. Sei $F_{m}{ }^{\prime}$ Bild von $F_{2}$ und $B, f_{m}^{1}, \ldots, f_{m}^{i}, \ldots, f_{m}^{k}, \lambda_{m}$ eine Bewertung von $F_{m}^{\prime}$, so dass $K_{m}\left(f_{m}, \lambda_{m}\right) \geqq \delta>0$ ist. Dann liegt der Wert von $F_{m}{ }^{\prime}$ in einem der beiden Intervalle

$$
I_{1}:[\delta, \lambda-\delta], \quad I_{2}:[\lambda+\delta, 2 \lambda-\delta] .
$$


BEweis. Wir wenden Induktion an. Für Formeln der Form $\varphi_{m}{ }^{\alpha_{1}}\left(x_{1}\right.$, $\left.\ldots, x_{n}\right)$ ist der Satz richtig, wie aus $K_{m}\left(f_{m}, \lambda_{m}\right) \geqq \delta>0$ und den vorangehenden Ausführungen folgt. Jetzt hat man Fallunterscheidungen zu machen.

(a) $F_{2}$ ist von der Form $A_{2} \wedge B_{2}$. Dann ist $F_{m}{ }^{\prime}$ von der Form $A_{m}{ }^{\prime} \wedge B_{m^{\prime}}$, wo $A_{m}{ }^{\prime}$ Bild von $A_{2}$ ist, $B_{m}{ }^{\prime}$ Bild von $B_{2}$. Auf $A_{m}{ }^{\prime}$ und $B_{m}{ }^{\prime}$ trifft der Satz aber nach Voraussetzung zu, d.h. der Wert $a$ von $A_{m}{ }^{\prime}$ liegt in $I_{1} \cup I_{2}$, desgleichen der Wert $b$ von $B_{m}{ }^{\prime}$. Der Wert von $A_{m}{ }^{\prime} \wedge B_{m}{ }^{\prime}$ ist aber gleich $\min (a, b)$, d.h. wieder eine $Z$ ahl aus $I_{1} \cup I_{2}$.

(b) $F_{2}$ ist von der Form $A_{2} \vee B_{2}$. Dann schliesst man gleich wie unter (a).

(c) $F_{2}$ ist von der Form $\neg A_{2}$. Dann ist $F_{m}$ von der Form $E\left(\Lambda_{m}{ }^{\prime}\right)-A_{m}{ }^{\prime}$. Auf $A_{m}{ }^{\prime}$ trifft der Satz aber zu, d.h. der Wert $a$ von $A_{m}{ }^{\prime}$ liegt in $I_{1} \cup I_{2}$. Der Wert von $F_{m}{ }^{\prime}$ ist aber gleich $2 \lambda_{m}-a$, d.h. wieder in $I_{1} \cup I_{2}$.

(d) $F_{2}$ ist von der Form $(E x) A_{2}(x)$, also $F_{m}^{\prime}$ von der Form $\operatorname{Max} A_{m}{ }^{\prime}(x)$, wobei $A_{m}{ }^{\prime}(x)$ Bild von $A_{2}(x)$ ist. Auf Grund der Induktionsvoraussetzung folgt, dass für jedes Individuum $\beta$ aus $B \operatorname{der}$ Wert $a_{\beta} \operatorname{von} A_{m}{ }^{\prime}(\beta)$ in $I_{1}$ oder $I_{2}$ liegt. Da aber $I_{1}$ ind $I_{2}$ abgeschlossene Intervalle sind, folgt, dass auch der Wert limsup $a_{\beta}$ von $\underset{x}{\operatorname{Max}} A_{m}^{\prime}(x)$ wieder in $I_{1} \cup I_{2}$ liegt.

(e) $F_{2}$ ist von der Form $(x) A_{2}(x)$. Dann schliesst man wieder wie unter (d), womit der Hilfssatz bewiesen ist.

Jetzt beweisen wir:

SATZ 1. Sei $F_{m}{ }^{\prime}$ Bild von $F_{2}$ und $B, f_{m}^{1}, \ldots, f_{m}{ }^{1}, \ldots, f_{m}{ }^{k}, \lambda_{m}$ eine Bewertung von $F_{m}{ }^{\prime}$, so dass $K_{m}\left(f_{m}, \lambda_{m}\right) \geqq \delta>0$ ist.

Setzt man

$$
\begin{aligned}
& f_{2}^{i}\left(\beta, \ldots, \beta_{n}\right)=\text { wahr }=\mathfrak{t} \text { für } f_{m}^{i}\left(\beta_{1}, \ldots, \beta_{n}\right) \in I_{2}, \\
& f_{2}^{i}\left(\beta_{1}, \ldots, \beta_{n}\right)=\text { falsch }=\mathfrak{f} \text { für } f_{m}^{i}\left(\beta_{1}, \ldots, \beta_{n}\right) \in I_{1},
\end{aligned}
$$

so folgt

$$
\begin{aligned}
& F_{2}\left(f_{2}{ }^{1}, \ldots, f_{2}{ }^{k}\right)=\mathfrak{t} \text { wenn } F_{m}{ }^{\prime}\left(f_{m}{ }^{1}, \ldots, f_{m}^{k}, \lambda_{m}\right) \in I_{2}, \\
& F_{2}\left(f_{2}{ }^{1}, \ldots, f_{2}{ }^{k}\right)=\mathfrak{f} \text { wenn } F_{m}\left(f_{m}{ }^{1}, \ldots, f_{m} k, \lambda_{m}\right) \in I_{1} .
\end{aligned}
$$

BeweIs. Man wendet wieder Induktion an. Für Formeln der Form $\varphi_{2}{ }^{\alpha_{1}}\left(x_{1}, \ldots, x_{n}\right)$ ist der Satz offenbar richtig. Jetzt hat man wieder Fallunterscheidungen vorzunehmen.

(a) $F_{2}$ ist von der Form $A_{2} \wedge B_{2}$. Dann ist $F_{m}{ }^{\prime}$ von der Form $A_{m}{ }^{\prime} \wedge B_{m^{\prime}}$, wo $A_{m}{ }^{\prime}$ Bild von $A_{2}$ ist, $B_{m}{ }^{\prime}$ Bild von $B_{2}$. Sei etwa der Wert $c$ von $F_{m}{ }^{\prime}$ in $I_{2}$. Dann gilt für den Wert $a$ von $A_{m}{ }^{\prime}$ und den Wert $b$ von $B_{m}{ }^{\prime}$ : $\min (a, b) \in I_{2}$, also $a \in I_{2}$ und $b \in I_{2}$. Auf Grund der Induktionsvoraussetzung ist der Satz aber richtig für $A_{2}$ und für $B_{2}$, d.h. $A_{2}\left(f_{2}{ }^{1}, \ldots, f_{2}{ }^{k}\right)=\mathfrak{t}$ und $B_{2}\left(f_{2}{ }^{1}, \ldots, f_{2}{ }^{k}\right)=\mathfrak{t}$, somit $F_{2}\left(f_{2}{ }^{1}, \ldots, f_{2}{ }^{k}\right)=\mathfrak{t}$, womit der Satz für diesen Fall bewiesen ist. Analog schliesst man im Fall $F_{m}{ }^{\prime}\left(f_{m}{ }^{1}, \ldots, f_{m}^{k}, \lambda_{m}\right) \in I_{1}$. 
(b) $F_{2}$ ist von der Form $A_{2} \vee B_{2}$. Dann schliesst man gleich wie unter (a).

(c) $F_{2}$ ist von der Form $\neg A_{2}$. Dann ist $F_{m}{ }^{\prime}$ von der Form $E\left(\Lambda_{m}{ }^{\prime}\right) \doteq A_{m}{ }^{\prime}$. Sei $c$ der Wert von $F_{m}{ }^{\prime}$, und sei $c \in I_{2}$. Für den Wert $a$ von $A_{m}{ }^{\prime}$ folgt dann $2 \lambda_{m}-a \dot{\epsilon} I_{2}$, d.h. $a \in I_{1}$. Dann ist aber $A_{2}\left(f_{2}^{1}, \ldots, f_{2} k\right)=\mathfrak{f}$, also $F_{2}\left(f_{2}{ }^{1}, \ldots, f_{2}{ }^{k}\right)=\mathrm{t}$, womit der Satz für diesen Fall bewiesen ist. Analog schliesst man im Fall $F_{m}{ }^{\prime}\left(f_{m}^{1}, \ldots, f_{m}^{k}, \lambda_{m}\right) \in I_{1}$.

(d) $F_{2}$ sei von der Form $(E x) A_{2}(x)$. Dann ist $F_{m}{ }^{\prime}$ von der Form $\operatorname{Max} A_{m}{ }^{\prime}(x)$, wobei $A_{m}{ }^{\prime}(x)$ Bild von $A_{2}(x)$ ist. Sei der Wert $c$ von $F_{m}^{\prime}$ in $I_{2}$. Dies ist gleichbedeutend mit $\limsup _{\beta} a_{\beta} \in I_{2}$, wo $a_{\beta} \operatorname{der}$ Wert von $A_{m}{ }^{\prime}(\beta)$ ist. Da $I_{1}$ und $I_{2}$ zwei disjunkte, abgeschlossene Intervalle sind, folgt, dass für mindestens ein $\beta_{0}$ der Wert $a_{\beta_{0}}$ in $I_{2}$ liegen muss. Auf Grund der Induktionsvoraussetzung folgt dann aber $A_{2}\left(\beta_{0}\right)=\mathrm{t}$, d.h. $(E x) A_{2}(x)$ erhält den Wert Wahr, womit der Satz für diesen Fall bewiesen ist. Analog schliesst man im Fall $c \in I_{1}$.

(e) $F_{2}$ ist von der Form $(x) A_{2}(x)$. Dann schliesst man gleich wie unter (d), womit der Satz bewiesen ist.

Aus diesem Satz folgt jetzt leicht:

SATZ 2. $F_{2}\left(\varphi_{2}{ }^{\alpha_{1}}, \ldots, \varphi_{2}{ }^{\alpha_{k}}\right)$ ist genau dann erfüllbar, wenn

$$
\left(F_{m}\left(\varphi_{m}^{\alpha_{1}}, \ldots, \varphi_{m}^{\alpha_{k}}, \Lambda_{m}\right) \doteq \Lambda_{m}\right) \wedge K_{m}\left(\varphi_{m}, \Lambda_{m}\right)
$$

erfüllbar grösser Null ist.

Bewers. (a) Sei $B, t_{m}^{1}, \ldots, f_{m}^{i}, \ldots, t_{m}^{k}, \lambda_{m}$ eine Bewertung derart, dass

$$
\min \left\{F_{m}\left(f_{m^{1}}, \ldots, f_{m}{ }^{k}, \lambda_{m}\right)-\lambda_{m}, K_{m}\left(f_{m}, \lambda_{m}\right)\right\} \geqq \delta>0
$$

ist. Dann ist speziell $K_{m}\left(f_{m}, \lambda_{m}\right) \geqq \delta>0$, d.h. die Voraussetzung von Satz 1 ist erfüllt. Dann liegt der Wert von $F_{m}$ in einem der beiden Intervalle $I_{1}:\left[\delta, \lambda_{m}-\delta\right], I_{2}:\left[\lambda_{m}+\delta, 2 \lambda_{m}-\delta\right]$. Auf die in Satz 1 angegebene Art gewinnt man eine Bewertung von $F_{2}$. Diese Bewertung ist so, dass aus $F_{m}\left(f_{m}, \lambda_{m}\right) \in I_{2}$ folgt $F_{2}\left(f_{2}\right)=\mathrm{t}$, aus $F_{m}\left(f_{m}, \lambda_{m}\right) \in I_{1}$ hingegen $F_{2}\left(f_{2}\right)=\mathfrak{f}$. Da aber auch $F_{m}\left(f_{m}, \lambda_{m}\right)-\lambda_{m} \geqq \delta>0$ ist, folgt $F_{m}\left(f_{m}, \lambda_{m}\right) \in I_{2}$, d.h. $F_{2}\left(f_{2}\right)=\mathrm{t}$, womit der erste Teil des Satzes bewiesen ist.

(b) Wenn $F_{2}$ erfüllbar ist, und etwa $B, f_{2}^{1}, \ldots, f_{2}^{i}, \ldots, f_{2}^{k}$ ein Erfüllungssystem ist, d.h. $F_{2}\left(f_{2}{ }^{1}, \ldots, f_{2}{ }^{k}\right)=\mathfrak{t}$, so setze man

$$
\begin{aligned}
& f_{m}^{i}\left(\beta_{1}, \ldots, \beta_{n}\right)=\frac{3}{5} \text { wenn } f_{2}^{i}\left(\beta_{1}, \ldots, \beta_{n}\right)=\mathfrak{t}, \\
& f_{m}^{i}\left(\beta_{1}, \ldots, \beta_{n}\right)=\frac{1}{5} \text { wenn } f_{2}^{i}\left(\beta_{1}, \ldots, \beta_{n}\right)=\mathfrak{f}
\end{aligned}
$$

und $\lambda_{m}=\frac{2}{5}$.

Dann zeigt man leicht mit Induktion $F_{m}\left(f_{m^{1}}, \ldots, f_{m}{ }^{k}, \frac{2}{5}\right)=\frac{3}{5}$ und $K_{m}\left(f_{m}, \lambda_{m}\right)=\frac{1}{5}$. Also hat mit dieser Bewertung die Formel

$$
\left(F_{m}\left(\varphi_{m}^{\alpha_{1}}, \ldots, \varphi_{m}^{\alpha_{k}}, \Lambda_{m}\right) \doteq \Lambda_{m}\right) \wedge K_{m}\left(\varphi_{m}, \Lambda_{m}\right)
$$

den Wert $\frac{1}{b}$, womit der Satz vollständig bewiesen ist. 
Jetzt soll noch die zweite Hilfssformel, $H_{m}\left(\varphi_{m}, \Lambda_{m}, \mathscr{H}_{m}\right)$, genauer untersucht werden. Seien $X_{m}$ und $Y_{m}$ zwei Aussagenvariablen, die von $\Lambda_{m}$ verschieden sind. $X_{m} * Y_{m}$ ist dann also die Formel

$$
\left(X_{m} \wedge Y_{m}\right) \vee\left(\left(E\left(\Lambda_{m}\right)-X_{m}\right) \wedge\left(E\left(\Lambda_{m}\right)-Y_{m}\right)\right)
$$

d.h. $X_{m} * Y_{m}$ ist nichts anderes als Bild der Formel

$$
\left(X_{2} \wedge Y_{2}\right) \vee\left(\neg X_{2} \wedge \neg Y_{2}\right)
$$

des zweiwertigen Kalküls, für die man oft die Abkürzung $X_{2} \equiv Y_{2}$ einführt. Sei jetzt $\xi_{1}, \xi_{2}, \lambda_{m}$ eine Bewertung der Formel $X_{m} * Y_{m}$ und sei $K_{m}\left(\xi, \lambda_{m}\right) \geqq \delta>0$. D.h. $\xi_{1}, \xi_{2}$ sind zwei Zahlen aus $[0,1]$, die durch die Forderung $K_{m}\left(\xi, \lambda_{m}\right) \geqq \delta>0$ auf die zwei Intervalle $I_{1}:\left[\delta, \lambda_{m}-\delta\right]$, $I_{2}:\left[\lambda_{m}+\delta, 2 \lambda_{m}-\delta\right]$ beschränkt sind.

Durch diese Bewertung erhält die Formel $X_{m} * Y_{m}$ den Wert

$$
\max \left\{\min \left(\xi_{1}, \xi_{2}\right), \min \left(2 \lambda_{m} \dot{-} \xi_{1}, 2 \lambda_{m} \dot{\left.-\xi_{2}\right)}\right\} .\right.
$$

Man sieht sofort, dieser Wert liegt genau dann in $\boldsymbol{I}_{\mathbf{2}}$, wenn die beiden Zahlen $\xi_{1}$ und $\xi_{2}$ aus dem gleichen Intervall stammen, d.h. zugleich $\xi_{1} \in I_{1}$ und $\xi_{2} \in I_{1}$ oder zugleich $\xi_{1} \in I_{2}$ und $\xi_{2} \in I_{2}$. Sei jetzt wieder $B, f_{m}^{1}, \ldots, f_{m}{ }^{\ell}, \ldots, f_{m}{ }^{k}$ eine Bewertung der Formel $F_{m}\left(\varphi_{m}{ }^{\alpha_{1}}, \ldots, \varphi_{m}{ }^{\alpha_{k}}, \Lambda_{m}\right)$, die der Formel $F_{2}\left(\varphi_{2}{ }^{\alpha_{1}}, \ldots, \varphi_{2}{ }^{\alpha_{k}}\right)$ zugeordnet ist. Ferner sei $K_{m}\left(f_{m}, \lambda_{m}\right) \geqq$ $\delta>0$. Die Individuen von $B$ teilen wir in Äquivalenzklassen. Wir sagen, $\alpha$ sei äquivalent $\beta$, wenn für alle Indizes $i, r$ und alle Elemente $\beta_{1}, \ldots, \beta_{r}, \beta_{r+2}, \ldots, \beta_{r}$ aus $B$ stets

$$
f_{m}^{i}\left(\beta_{1}, \ldots, \beta_{r}, \alpha, \beta_{r+2}, \ldots, \beta_{n}\right) * f_{m}^{i}\left(\beta_{1}, \ldots, \beta_{r}, \beta, \beta_{r+2}, \ldots, \beta_{n}\right) \in I_{2}
$$

gilt, wo $n$ die Stellenzahl von $\varphi_{2} \alpha_{i}$ (und somit von $f_{m}{ }^{\imath}$ ) ist. Offenbar ist $\alpha$ äquivalent $\beta$ genau dann, wenn

$$
\min \left\{D_{m}\left(f_{m}^{1}, \alpha, \beta, \lambda_{m}\right), \ldots, D_{m}\left(f_{m}{ }^{k}, \alpha, \beta, \lambda_{m}\right)\right\} \in I_{2}
$$

ist. Definiert man wieder, wie oben angegeben, die Prädikate $f_{2}{ }^{t}$ wie folgt,

$$
\begin{aligned}
& f_{2}^{i}\left(\beta_{1}, \ldots, \beta_{n}\right)=\mathfrak{t} \text { wenn } f_{m}^{i}\left(\beta_{1}, \ldots, \beta_{n}\right) \in I_{2}, \\
& f_{2}\left(\beta_{1}, \ldots, \beta_{n}\right)=\mathfrak{f} \text { wenn } f_{m}^{i}\left(\beta_{1}, \ldots, \beta_{n}\right) \in I_{1},
\end{aligned}
$$

so sieht man, dass äquivalente Elemente solche sind, die bezüglich der Prädikate $f_{2}$ miteinander identifiziert werden können, d.h. als gleich betrachtet werden können.

Nun können wir folgenden Satz beweisen.

SAT2 3. Aus $H_{m}\left(f_{m}, \lambda_{m}, h_{m}\right)>0$ folgt, dass es nur endlich viele Äquivalenzklassen gibt.

BEwEIs. Wir nehmen an, das Gegenteil sei der Fall. D.h. es gibt ein 
$\varepsilon>0$, so dass $H_{m}\left(f_{m}, \lambda_{m}, h_{m}\right)=\varepsilon$ ist. D.h.

$$
\begin{aligned}
\operatorname{Min}_{x, y}\left\{\operatorname { m a x } \left(\left(\min \left(D_{m}\left(f_{m}^{1}, x, y, \lambda_{m}\right), \ldots, D_{m}\left(f_{m}^{k}, x, y, \lambda_{m}\right)\right)\right.\right.\right. & \left.-\lambda_{m}\right), \\
& \left.\left.\left|h_{m}(x)-h_{m}(y)\right|\right)\right\}=\varepsilon
\end{aligned}
$$

wo $h_{m}(x)$ die Funktion ist, die für $\mathscr{H}_{m}$ eingesetzt wird.

Für alle Elemente $\alpha, \beta$ aus $B$ gilt also

$$
\max \left\{\left(\min \left(D_{m}\left(f_{m}^{1}, \alpha, \beta, \lambda_{m}\right), \ldots, D_{m}\left(f_{m}^{k}, \alpha, \beta, \lambda_{m}\right)\right)-\lambda_{m}\right),\right.
$$

$$
\left.\left|h_{m}(\alpha)-h_{m}(\beta)\right|\right\} \geqq \varepsilon .
$$

Zwei Elemente $\alpha, \beta$ sind aber genau dann nichtäquivalent, wenn der Wert von

$$
\min \left(D_{m}\left(f_{m}^{1}, \alpha, \beta, \lambda_{m}\right), \ldots, D_{m}\left(f_{m}{ }^{k}, \alpha, \beta, \lambda_{m}\right)\right)
$$

in $I_{1}$ liegt, d.h. wenn der Wert von

$$
\min \left(D_{m}\left(f_{m}{ }^{1}, \alpha, \beta, \lambda_{m}\right), \ldots, D_{m}\left(f_{m}^{i}, \alpha, \beta, \lambda_{m}\right)\right)-\lambda_{m}
$$

gleich 0 ist. Dann muss aber

$$
\left|h_{m}(\alpha)-h_{m}(\beta)\right| \geqq \varepsilon
$$

sein, und dies ist gleichbedeutend mit

$$
\left|h_{m}(\alpha)-h_{m}(\beta)\right| \geqq \varepsilon .
$$

Sei jetzt $\beta_{1}, \ldots, \beta_{s}, \ldots$ eine abzählbare Menge von nichtäquivalenten Individuen. Dann gilt für alle $i \neq k$

$$
\left|h_{m}\left(\beta_{i}\right)-h_{m}\left(\beta_{k}\right)\right| \geqq \varepsilon .
$$

Dies führt aber sofort zum Widerspruch mit der Tatsache, dass eine unendliche, beschränkte Punktmenge einen Häufungspunkt hat. Damit ist die Behauptung bewiesen.

Daraus ergibt sich jetzt leicht:

SATZ 4. $F_{2}\left(\varphi_{2}{ }^{\alpha_{1}}, \ldots, \varphi_{2}{ }^{\alpha_{k}}\right)$ ist genau dann erfüllbar in einem endlichen Individuenbereich, wenn

$$
\left(F_{m}\left(\varphi_{m}{ }^{\alpha_{1}}, \ldots, \varphi_{m}^{\left.\alpha_{k}, \Lambda_{m}\right)}-\Lambda_{m}\right) \wedge K_{m}\left(\varphi_{m}, \Lambda_{m}\right) \wedge H_{m}\left(\varphi_{m}, \Lambda_{m}, \mathscr{H}_{m}\right)\right.
$$

erfüllbar grösser Null ist.

BewEIs. (a) Wir beweisen zuerst: ist $\left(F_{m} \doteq \Lambda_{m}\right) \wedge K_{m} \wedge H_{m}$ erfüllbar grösser Null, so ist $F_{2}$ erfüllbar im Endlichen.

Sei also $B, f_{m}{ }^{1}, \ldots, f_{m}{ }^{k}, \lambda_{m}, h_{m}$, ein Bewertungssystem, so dass

$$
\min \left\{\left(F_{m}\left(f_{m}{ }^{1}, \ldots, f_{m}{ }^{k}, \lambda_{m}\right)-\lambda_{m}\right), K_{m}\left(f_{m}, \lambda_{m}\right), H_{m}\left(f_{m}, \lambda_{m}, h_{m}\right)\right\} \geqq \delta>0
$$

ist. Dann ist speziell $\min \left(\left(F_{m}\left(f_{m}, \lambda_{m}\right)-\lambda_{m}\right), K_{m}\left(f_{m}, \lambda_{m}\right)\right) \geqq \delta>0$. Auf Grund von Satz 2 gewinnt man dann aber für $F_{2}$ ein Erfüllungssystem, 
indem man für die $\varphi_{2}{ }^{\alpha_{i}}$ die Prädikate $f_{2}{ }^{i}$ einsetzt, die ja wie folgt definiert sind:

$$
\begin{aligned}
& f_{2}^{i}\left(\beta_{1}, \ldots, \beta_{n}\right)=\mathrm{t} \text { wenn } f_{m}^{i}\left(\beta_{1}, \ldots, \beta_{n}\right) \in I_{2}, \\
& f_{2}^{i}\left(\beta_{1}, \ldots, \beta_{n}\right)=\mathfrak{f} \text { wenn } f_{m}^{i}\left(\beta_{1}, \ldots, \beta_{n}\right) \in I_{1},
\end{aligned}
$$

wobei $\beta_{1}, \beta_{2}, \ldots, \beta_{n}$ Individuen aus $B$ sind und $I_{1}, I_{2}$ die Intervalle $\left[\delta, \lambda_{m}-\delta\right]$ bzw. $\left[\lambda_{m}+\delta, 2 \lambda_{m}-\delta\right]$. Wegen $K_{m}\left(f_{m}, \lambda_{m}\right) \geqq \delta>0$ und $H_{m}\left(f_{m}, \lambda_{m}, h_{m}\right) \geqq \delta>0$ folgt aus Satz 3 , dass es nur endlich viele Äquivalenzklassen gibt. $\mathrm{Da}$ für das Erfüllungssystem von $F_{2}$ Elemente aus gleicher Äquivalenzklasse einander gleichgesetzt werden können, bedeutet das, dass $F_{2}$ in einem endlichen Individuenbereich erfüllbar ist.

(b) Sei $F_{2}$ in einem endlichen Individuenbereich erfüllbar und $N$ sei die Zahl der Individuen. Wir setzen

$$
\begin{aligned}
& f_{m}^{i}\left(\beta_{1}, \ldots, \beta_{n}\right)=\frac{3}{5} \text { wenn } f_{2}^{i}\left(\beta_{1}, \ldots, \beta_{n}\right)=\mathfrak{t} \\
& f_{m}^{i}\left(\beta_{1}, \ldots, \beta_{n}\right)=\frac{1}{5} \text { wenn } f_{2}^{i}\left(\beta_{1}, \ldots, \beta_{n}\right)=\mathfrak{f} .
\end{aligned}
$$

Sind $\beta_{1}, \beta_{2}, \ldots, \beta_{N}$ die Individuen von $B$, so sei ferner $h_{m}\left(\beta_{i}\right)=\frac{i}{5 N}$ und $\lambda_{m}=\frac{2}{5}$. Wie leicht ersichtlich, ist dann der Wert von $\left(F_{m}-\Lambda_{m}\right) \wedge K_{m}$ gleich $\frac{1}{5}$. Ferner seien $\alpha$ und $\beta$ zwei Elemente aus $B$. Sind $\alpha$ und $\beta$ ä.quivalent, so hat der Ausdruck

$$
\max \left\{\left(\min \left(D_{m}\left(f_{m}{ }^{1}, \alpha, \beta, \frac{2}{5}\right), \ldots, D_{m}\left(f_{m}{ }^{k}, \alpha, \beta, \frac{2}{5}\right)\right)-\frac{2}{5}\right),\left|h_{m}(\alpha)-h_{m}(\beta)\right|\right\}
$$

den Wert $\frac{1}{5}$, weil das erste Argument den Wert $\frac{1}{5}$ hat. Sind hingegen $\alpha$ und $\beta$ nichtäquivalent, so ist der Wert des ersten Argumentes Null, während der Wert des zweiten Argumentes $\geqq \frac{1}{5 N}$ ist. Also gilt

$$
\underset{x, y}{\liminf } \max \left\{\left(\min \left(D_{m}\left(f_{m}{ }^{1}, x, y, \frac{2}{5}\right), \ldots\right)-\frac{2}{5}\right),\left|h_{m}(x)-h_{m}(y)\right|\right\}=\frac{1}{5 N},
$$

d.h. $\min \left(F_{m}-\frac{2}{5}, K_{m}, H_{m}\right)=\frac{1}{5 N}$, womit der Satz bewiesen ist.

Schliesslich benötigen wir noch einen Satz, dessen Beweis hier nur skizziert werden soll, da er sich unmittelbar aus bekannten Tatsachen ergibt.

SATz 5. Die Menge der Formeln des zweiwertigen Prädikatenkalküls, die nicht im Endlichen erfüllbar sind, ist nicht rekursiv aufzählbar.

BEWEIS. Die Menge der Formeln, die im Endlichen erfüllbar sind, ist nicht rekursiv, wie bekannt [7]. Andererseits ist sie rekursiv aufzählbar, wie leicht zu sehen. Dann ist die Komplementärmenge, d.h. die Menge der Formeln, die nicht im Endlichen erfüllbar sind, nicht rekursiv aufzählbar da sonst nach einem bekannten Satz [2] beide Mengen schon rekursiv wären. 
Sei jetzt eine geeignete Gödelnummerierung der Formeln des zweiwertigen Kalküls eingeführt, desgleichen eine Gödelnummerierung der Formeln des unendlichwertigen Kalküls. Nun sei $F_{2}\left(\varphi_{2}{ }^{\alpha_{1}}, \ldots, \varphi_{2}{ }^{\alpha_{k}}\right)$ eine Formel des zweiwertigen Kalküls und $a$ ihre Gödelnummer. Dann gibt es, wie leicht zu sehen, aber umständlich zu beweisen; eine rekursive Funktion $m(x)$, deren Wert $m(a)$ die Gödelnummer der Formel

$$
\left(F_{m}\left(\varphi_{m}^{\alpha_{1}}, \ldots, \varphi_{m}^{\alpha_{k}}, \Lambda_{m}\right)-\Lambda_{m}\right) \wedge K_{m}\left(\varphi_{m}, \Lambda_{m}\right) \wedge H_{m}\left(\varphi_{m}, \Lambda_{m}, \mathscr{H}_{m}\right)
$$

ist. Jetzt folgt sofort:

SATZ 6. Die Menge der Formeln des unendlichwertigen Kalküls, die identisch Null sind, ist nicht rekursiv aufzählbar.

BeweIs. Wir nehmen an, das Gegenteil sei der Fall. Dann existiert ein rekursives Prädikat $S(u, v)$ mit der Eigenschaft: die Formel $G_{m}$ des unendlichwertigen Kalküls ist genau dann identisch Null, wenn für die zugehörige Gödelnummer $g$ die Aussage $(E v) S(g, v)$ richtig ist. Mit Hilfe von Satz 4 folgt dann aber: eine Formel $F_{2}$ des zweiwertigen Kalküls ist genau dann nicht im Endlichen erfüllbar, wenn für die zugehörige Gödelnummer $a$ die Aussage $(E v) S(m(a), v)$ richtig ist. Dies steht aber im Widerspruch zu Satz 5.

Mit Satz 6 haben wir zugleich bewiesen, dass die Menge der Formeln identisch Null nicht axiomatisierbar ist. Wie aus Satz 2 hervorgeht, ist auch die Menge der Formeln erfüllbar grösser Null nicht rekursiv aufzählbar und man beweist leicht, dass dies auch auf die Menge der Formeln, die erfüllbar 1 sind, zutrifft. Hingegen lässt sich zeigen, dass die Menge der Formeln, die nie den Wert Null annehmen, d.h. identisch grösser Null sind, rekursiv aufzählbar ist. Man zeigt dies, indem man mit Hilfe von Stetigkeitsargumenten beweist, dass sich der Wert, den eine Formel durch eine Bewertung erhält, beliebig genau approximieren lässt durch Bewertungssysteme, die nur Werte auf den Punkten $0, \frac{1}{n}, \frac{2}{n}, \ldots, \frac{n-1}{n}, 1$ annehmen $(n=1,2, \ldots)$.

Durch ähnliche Argumente lässt sich beweisen: die Menge der Formeln identisch Null gestattet eine Darstellung der Form $(u)(E v) S(x, u, v)$ mit rekursivem $S$. Ungelöst ist das Problem, ob diese Menge sich auch in der Form $(E u)(v) R(x, u, v)$ darstellen lässt. ${ }^{1}$

Herrn Prof. Specker sei herzlich gedankt für die Anregung zu dieser Arbeit und sein stetes Interesse, das er ihr entgegengebrachte.

1 (Added June 2, 1961.) In der Zwischenzeit konnte der Autor zeigen, dass sich die Menge der Formeln identisch Null nicht in der Form $(E u)(v) R(x, u, v)$ darstellen lässt. Der Beweis ergibt sich leicht mit den hier entwickelten Methoden. 


\section{LITERATURVERZEICHNIS}

[1] C. C. Chang, Algebraic analysis of many-valued logics, Transactions of the American Mathematical Soclety, Bd. 88 (1958), S. 467-490.

[2] S. C. KLEENE, Introduction to metamathematics, Amsterdam und Groningen (Noordhoff), New York und Toronto (Van Nostrand), 1952.

[3] J. EUKASIEWICZ und A. TARSKI, Untersuchungen über den Aussagenkalkül. Comptes rendus des séances de la Société des Sclences et des Lettres de Varsovie, Classe III, Bd. 23 (1930), S. 30-50.

[4] A. Rose und B. Rosser, Fragment of many-valued statement calculi, Transactions of the American Mathematical Society, Bd. 87 (1958), S. 1-53.

[5] J. BARKLEY ROSSER, Axiomatization of infinite-valued logics, Loglque et Analyse, Dezember 1960, S. 135-153.

[6] J. D. RUTLEDGE, On the definition of an infinitely-many-valued predicate calculus, dieses Journal, Bd. 24 (1960), S.

[7] B. A. TRAHTÉNBROT, Névozmožnost' algorifma dlá problémy razrésimosti na koné̌nyh klassah (Unmöglichkeit eines Algorithmus für die Entscheidungsproblem in endlichen Klassen), Doklady Akadémii Nauk SSSR, n. R., Bd. 70 (1950), S. 569-572.

EIDGENÖSSISCHE TECHNISCHE HOCHSCHULE, ZÜRICH. 